Fousseni FÉTÉKÉ⿱ Jérôme PERIN ${ }^{1}$ Adeline FAYOLLE ${ }^{1}$ Kasso DAINOU ${ }^{2}$

Nils BOURLAND ${ }^{3}$

Yao Lambert KouAdIO 4

Serge Jean Joel MONEYE ${ }^{5}$

Carlos-Claude BEKONO ${ }^{6}$

Mbona Yem LiBoum ${ }^{6}$

Jean-Louis DoucET ${ }^{1}$

Philippe LeJEUnE ${ }^{1}$

${ }^{1}$ Université de Liège Gembloux Agro-Bio Tech

Département BIOSE

Passage des Déportés 2

5030 Gembloux

Belgique

${ }^{2}$ Nature Plus ASBL

Rue Bourgmestre Gilisquet 57 1457 Walhain-Saint-Paul Belgique

${ }^{3}$ Musée royal

de l'Afrique centrale

Service de biologie du bois 3080 Tervuren

Belgique

4 Université Nangui Abrogoua Unité de formation

et recherche des Sciences

de la Nature

01 BP 4403

Abidjan

Côte d'Ivoire

5 Université de Kinshasa

ERAIFT

BP 15173

République démocratique du Congo

${ }^{6}$ Université de Dschang Faculté d'agronomie et des sciences agricoles (FASA)

BP 96, Dschang

Cameroun

\section{Modéliser la croissance de quatre essences pour améliorer la gestion forestière au Cameroun}

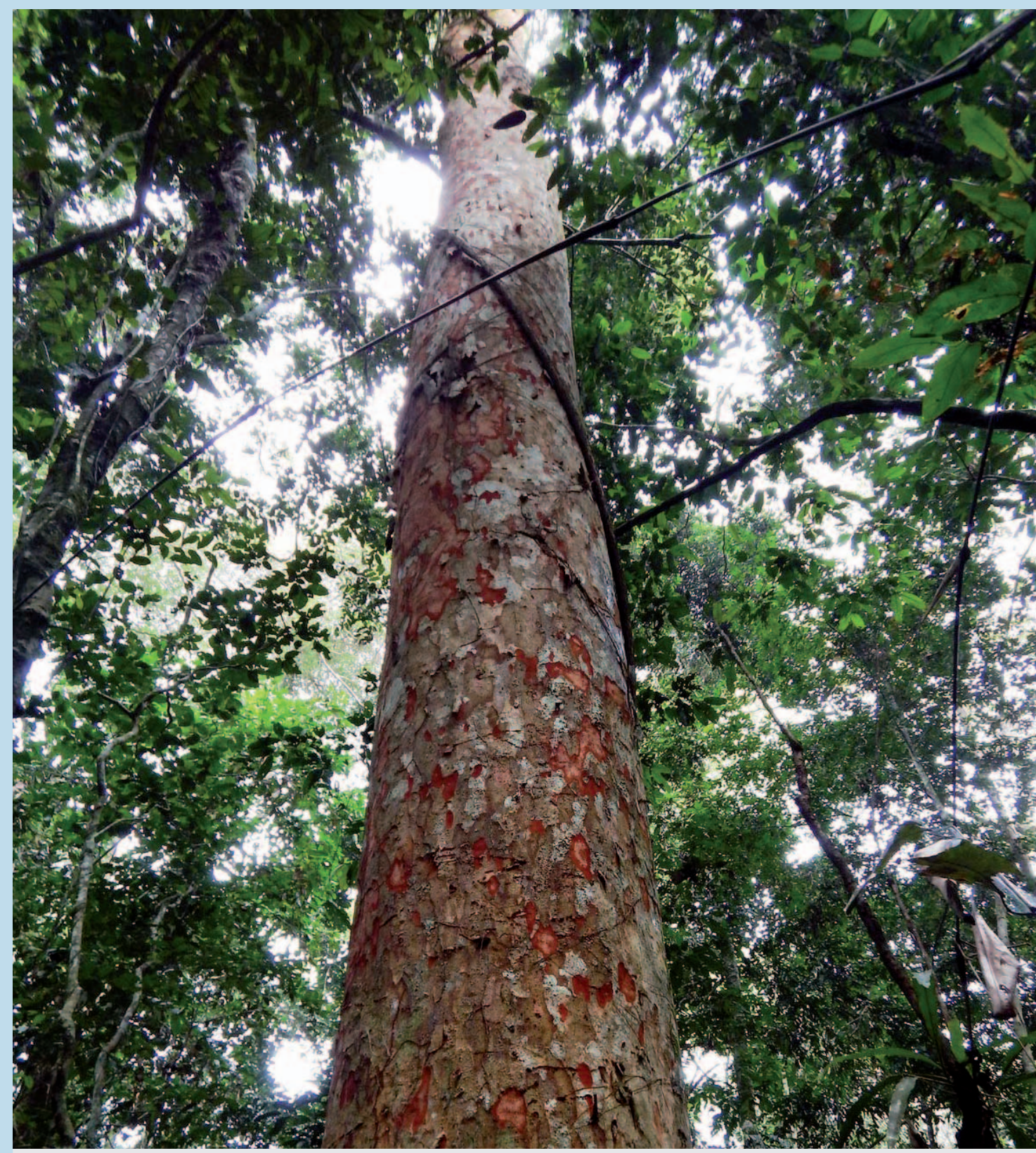

Photo 1.

Pied d'assamela (Pericopsis elata).

Photo N. Bourland. 


\section{RÉSUMÉ}

\section{MODÉLISER LA CROISSANCE DE QUATRE ESSENCES POUR AMÉLIORER LA GESTION FORESTIĖRE AU CAMEROUN}

En Afrique centrale, l'aménagement forestier repose sur une méthode de simulation de la production forestière peu précise, susceptible d'hypothéquer la durabilité de l'exploitation. L'objectif de cette étude est d'améliorer la prédiction de la croissance pour quatre essences commerciales importantes en Afrique centrale. Le dispositif d'étude a été installé dans le Sud-Est du Cameroun et comporte 387 arbres : 136 assamela (Pericopsis elata), 54 moabi (Baillonella toxisperma), 124 sapelli (Entandrophragma cylindricum), et 73 tali (Erythrophleum suaveolens). Le diamètre a été mesuré régulièrement entre 2009 et 2012. L'intensité de la compétition a été quantifiée à l'échelle d'une placette circulaire de $20 \mathrm{~m}$ de rayon installée autour de chaque arbre focal. Les caractéristiques topographiques et hydrologiques ont été estimées à l'aide d'un système d'information géographique. La croissance a été modélisée en intégrant le diamètre des arbres et les conditions de croissance (indice de compétition et environnement local). Les valeurs d'accroissement utilisées actuellement au Cameroun ne sont pas différentes des valeurs observées en forêt non perturbée par l'exploitation forestière pour l'assamela, le moabi et le tali, mais surestiment la croissance de $20 \%$ pour le sapelli. Un modèle log-normal intégrant le diamètre des arbres et l'intensité de la compétition permet d'estimer l'accroissement des quatre essences avec un coefficient de détermination $\left(R^{2}\right)$ variant de 0,092 pour l'assamela à 0,47 pour le moabi. Les variables topographiques et hydrologiques ne permettent pas d'améliorer significativement la qualité des prédictions. Ces modèles peuvent être utilisés pour prédire l'accroissement à partir des données d'inventaires forestiers d'aménagement tels qu'ils sont réalisés en Afrique centrale, en vue d'améliorer la définition de paramètres de gestion tels que le diamètre minimum d'exploitation ou le taux de reconstitution pour les quatre essences étudiées.

Mots-clés : Pericopsis elata, Baillonella toxisperma, Entandrophragma cylindricum, Erythrophleum suaveolens, modèle de croissance, gestion forestière, prédiction, diamètre minimum d'exploitation, Cameroun.

\section{ABSTRACT}

\section{MODELLING GROWTH IN FOUR SPECIES TO IMPROVE FOREST MANAGEMENT IN CAMEROON}

In Central Africa, forest planning relies on a method of forest production simulation that lacks precision and is liable to compromise logging sustainability. The aim of this study was to improve growth forecasts for four of the main commercial species in Central Africa. A survey was set up in south-eastern Cameroon, covering 387 trees: 136 Assamela (Pericopsis elata), 54 Moabi (Baillonella toxisperma), 124 Sapelli (Entandrophragma cylindricum) and 73 Tali (Erythrophleum suaveolens). Their diameter was measured regularly from 2009 to 2012. Competition intensity was quantified on the scale of a circular plot with a radius of $20 \mathrm{~m}$ around each target tree. Topographic and hydrological characteristics were estimated with the aid of a geographic information system. Growth was modelled on the basis of tree diameter and growth conditions (competition index and local environment). The tree growth figures currently used in Cameroon are identical to those observed in forests undisturbed by logging in the case of Assamela, Moabi and Tali, but they overestimate the growth of Sapelli by $20 \%$. A log-normal model incorporating tree diameter and competition intensity produced growth estimations for the four species with a determination coefficient $\left(R^{2}\right)$ ranging from 0.092 for Assamela to 0.47 for Moabi. The topographic and hydrological variables did not significantly improve the quality of the forecasts. These models may be used to forecast growth on the basis of data from forest inventories as currently drawn up for planning purposes in Central Africa, in order to improve the definition of management parameters for the four species studied, such as minimum logging diameters or regeneration rates.

Keywords: Pericopsis elata, Baillonella toxisperma, Entandrophragma cylindricum, Erythrophleum suaveolens, growth model, forest management, forecast, minimum logging diameter, Cameroon.

\section{RESUMEN}

\section{MODELIZAR EL CRECIMIENTO DE CUATRO ESPECIES PARA MEJORAR EL MANEJO FORESTAL EN CAMERÚN}

La ordenación forestal en África Central se basa en un método de simulación de la producción forestal poco preciso y que puede comprometer la sostenibilidad del aprovechamiento. El objetivo de este estudio es mejorar la predicción del crecimiento de cuatro importantes especies comerciales de África Central. El diseño del estudio se estableció en el sudeste de Camerún e incluye 387 árboles: 136 Assamela (Pericopsis elata), 54 Moabi (Baillonella toxisperma), 124 Sapelli (Entandrophragma cylindricum) y 73 Tali (Erythrophleum suaveolens). Se midió regularmente el diámetro entre 2009 y 2012. Se cuantificó la intensidad de la competencia en una parcela circular de $20 \mathrm{~m}$ de radio trazada alrededor de cada árbol focal. Las características topográficas e hidrológicas se calcularon mediante un sistema de información geográfica. El crecimiento se modelizó integrando el diámetro de los árboles y las condiciones de crecimiento (índice de competencia y ambiente local). Los valores de incremento actualmente empleados en Camerún no son diferentes de los observados en bosques no perturbados por el aprovechamiento forestal en Assamela, Moabi y Tali, pero sobreestiman el crecimiento del Sapelli en un $\mathbf{2 0} \%$. Un modelo lognormal que integra el diámetro de los árboles y la intensidad de la competencia permite evaluar el incremento de las cuatro especies con un coeficiente de determinación $\left(R^{2}\right)$ que varía entre 0,092 para Assamela a 0,47 para Moabi. Las variables topográficas e hidrológicas no permiten mejorar significativamente la calidad de las predicciones. Estos modelos pueden utilizarse para predecir el incremento, basándose en los datos de inventarios forestales de ordenación que se realizan actualmente en África Central, con el objetivo de mejorar la definición de parámetros de manejo como el diámetro mínimo de aprovechamiento o la tasa de restauración de las cuatro especies estudiadas.

Palabras clave: Pericopsis elata, Baillonella toxisperma, Entandrophragma cylindricum, Erythrophleum suaveolens, modelo decrecimiento, manejo forestal, predicción, diámetro mínimo de aprovechamiento, Camerún. 


\section{Introduction}

Les forêts denses humides du bassin du Congo couvrent environ 189 millions d'hectares (De Wasseige et al., 2012) et abritent une très grande diversité biologique. Elles fournissent des services écologiques et surtout économiques importants dont l'exploitation commerciale du bois d'œuvre. Environ $24 \%$ de la superficie de ces forêts est attribuée, sous forme de concessions, à des sociétés privées qui exploitent plus de huit millions de mètres cubes de bois d'œuvre chaque année (Bayol et al., 2012).

Depuis les années 1990, le concept d'aménagementexploitation a été érigé comme outil de référence de la gestion des forêts d'Afrique centrale (Fargeot et al., 2004). Ce concept, qui vise à assurer une production forestière soutenue et durable, est basé sur deux principes : garantir la reconstitution du stock exploitable et planifier la récolte dans l'espace et le temps afin de garantir une régularité de l'approvisionnement en bois. Le principe de reconstitution du stock exploitable repose sur la prise en compte d'un taux de reconstitution défini comme le ratio du stock exploitable futur sur le stock exploitable initial, le stock exploitable étant lui-même lié à la définition d'un diamètre seuil (DME pour diamètre minimum d'exploitation) en deçà duquel les arbres ne peuvent être récoltés (Durrieu de Madron et Forni, 1997). La vitesse avec laquelle le stock exploitable est reconstitué après récolte dépend directement de la vitesse de croissance et du taux de mortalité entre deux exploitations. Ces deux paramètres dynamiques doivent être connus de manière aussi précise que possible pour que la méthode fournisse des résultats pertinents (Picard et al., 2008). Si l'on considère plus particulièrement l'accroissement, la formulation proposée par Durrieu de Madron et Forni (1997) repose sur un accroissement moyen considéré comme constant sur toute la gamme de diamètres. Plusieurs travaux font cependant clairement apparaître une dépendance de la croissance vis-à-vis du diamètre des arbres (Gourlet-Fleury, 1998 ; Hérault et al., 2011 en Guyane française ; Obiang et al., 2012 et 2013 au Gabon). En outre, les valeurs d'accroissement utilisées dans les plans d'aménagement sont des valeurs de référence imposées par l'administration forestière, dont l'origine et la représentativité au plan statistique (domaine d'application, variabilité résiduelle, absence de biais) sont rarement documentées.

À ce jour, des modèles de croissance ont été développés pour un nombre très limité d'espèces exploitées en Afrique centrale. C'est le cas de l'azobé, Lophira alata Banks ex C.F. Gaertn., et de l'okoumé, Aucoumea klaineana Pierre, au Gabon (Obiang et al., 2012, 2013). Ces modèles intègrent d'une part les caractéristiques intrinsèques des arbres (diamètre) et d'autre part la compétition exercée par les arbres voisins (Moravie et al., 1997 ; Gourlet-Fleury et Houllier, 2000 ; Obiang et al., 2012, 2013). Les variables environnementales traduisant la potentialité du site sont fréquemment utilisées pour modéliser la croissance des peuplements irréguliers mélangés en région tempérée (Lejeune, 1996) ou boréale (Pokharel et Dech, 2012). Leur utilisation est par contre beaucoup plus rare dans les régions tropicales, sans doute en raison du manque de données appropriées.
Le but de la présente étude est de développer des modèles de la croissance des arbres intégrant le diamètre et les conditions de croissance (indice de compétition et environnement local) pour quatre essences largement exploitées pour le bois d'œuvre dans les forêts semi-caducifoliées du Sud-Est du Cameroun : l'assamela, Pericopsis elata (Harms) van Meeuwen (Fabaceae), le moabi, Baillonella toxisperma Pierre (Sapotaceae), le sapelli, Entandrophragma cylindricum (Sprague) Sprague (Meliaceae), et le tali, Erythrophleum suaveolens (Guillemin \& Perrottet) Brenan (Fabaceae, Caesalpioniodeae). Ces quatre essences contribuent à plus de $25 \%$ de la production moyenne annuelle du pays (De Wasseige et al., 2012). Actuellement, les valeurs de référence pour la croissance diamétrique de ces espèces imposées par l'administration forestière camerounaise dans le calcul des taux de reconstitution sont de $0,5 \mathrm{~cm} /$ an pour le sapelli et de 0,4 cm/an pour l'assamela, le moabi et le tali. Les données utilisées pour calculer ces moyennes proviennent de dispositifs d'étude installés dans quelques sites en Afrique centrale et de l'Ouest (Détienne et al., 1998) mais leur variabilité reste imprécise. II apparaît donc nécessaire de tester dans un premier temps la validité de ces valeurs et d'évaluer ensuite le gain de précision que fourniraient des modèles de croissance dépendant du diamètre et/ou de l'intensité de la compétition et des conditions environnementales locales.

\section{Matériel et méthode}

\section{Site d'étude}

Le site d'étude regroupe six unités forestières d'aménagement (UFA 10-030, 10-031, 10-039, 10-041, 10-042 et 10-044) gérées par la société d'exploitation forestière certifiée Pallisco. Il est situé dans le Sud-Est du Cameroun, entre $3^{\circ} 01^{\prime}$ et $3^{\circ} 44^{\prime}$ de latitude Nord et $13^{\circ} 20^{\prime}$ et $14^{\circ} 31^{\prime}$ de longitude Est (figure 1), dans une zone de transition entre les forêts sempervirentes et les forêts semi-caducifoliées (Letouzey, 1985). L'altitude de la zone varie entre 600 et $760 \mathrm{~m}$, avec un relief faiblement accidenté. Le climat est de type équatorial, avec des précipitations annuelles moyennes de1 $458 \mathrm{~mm}$ pendant la période de suivi de la croissance et de $1419 \mathrm{~mm}$ pour la période de 1983 à 2014 (extrait de la base de données ARCv2 ; Novella et Thiaw, 2012). La température annuelle moyenne est de $24^{\circ} \mathrm{C}$. Les sols sont de type ferrallitique pour les forêts de terre ferme.

\section{Espèces étudiées}

\section{L’assamela : Pericopsis elata (photo 1)}

Sa distribution se limite aux forêts denses humides semi-caducifoliées s'étendant de la Côte d'Ivoire à la République démocratique du Congo (RDC). Il s'agit d'une espèce héliophile pionnière anémochore (Hall et Swaine, 1981 ; Bourland et al., 2012), supportant les variations de la teneur en eau du sol, préférant les sols fertiles, riches en magnésium, phosphore ${ }^{1}$. L'accroissement diamétrique varie entre

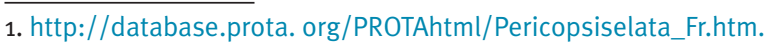




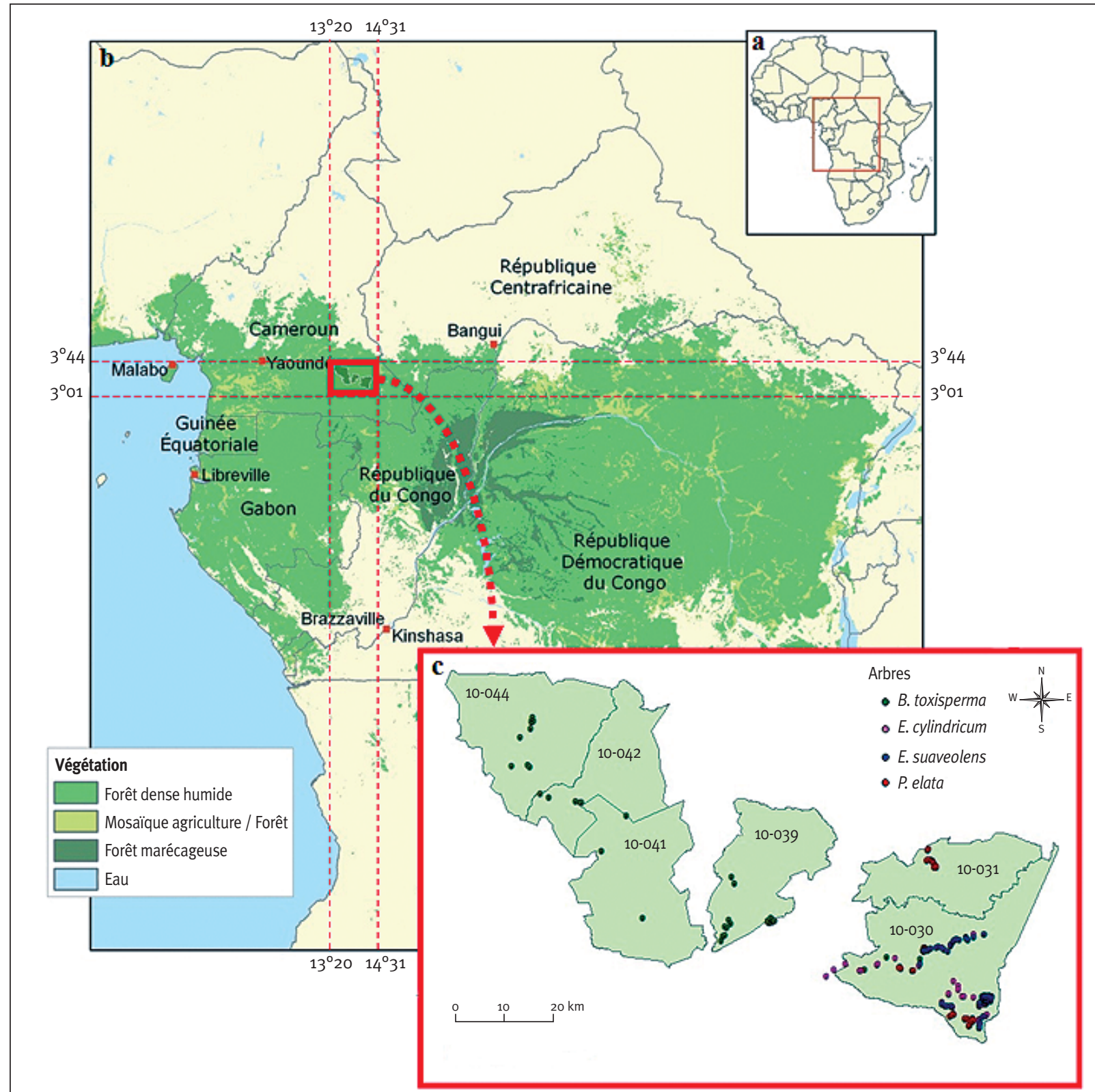

Figure 1.

Localisation du site d'étude en Afrique (a) et au Cameroun (b), et distribution des 387 arbres suivis pour la croissance au sein des unités forestières d'aménagement (UFA) exploitées par la société Pallisco (c). Les principaux types de végétation de l'Afrique centrale sont indiqués (source : WRI, 2009).

0,32 et 0,45 cm/an au Cameroun (Bourland et al., 2012). La densité moyenne du bois est de l'ordre de 0,57 à $0,71 \mathrm{~g} / \mathrm{cm}^{3}$ (Zanne et al., 2009).

\section{Le moabi : Baillonella toxisperma (photo 2)}

Sa distribution est limitée aux zones de forêt sempervirente et semi-caducifoliée de basse altitude $(<500 \mathrm{~m})$ et s'étend du Sud du Nigeria au Gabon, au Congo et au SudOuest de la RDC (Debroux, 1998). Cette espèce est hélio- phile non pionnière, zoochore et ne supporterait pas les sols trop drainants (Doucet et al., 2009). L'accroissement moyen peut atteindre 0,95 cm/an (Debroux, 1998). La densité de son bois est élevée et varie entre 0,82 et $0,94 \mathrm{~g} / \mathrm{cm}^{3}$ (Zanne et al., 2009).

\section{Le sapelli : Entandrophragma cylindricum (photo 3)}

Il présente une large distribution dans la zone forestière allant de la Sierra Leone à l'Ouganda, et du Sud de la RDC à 


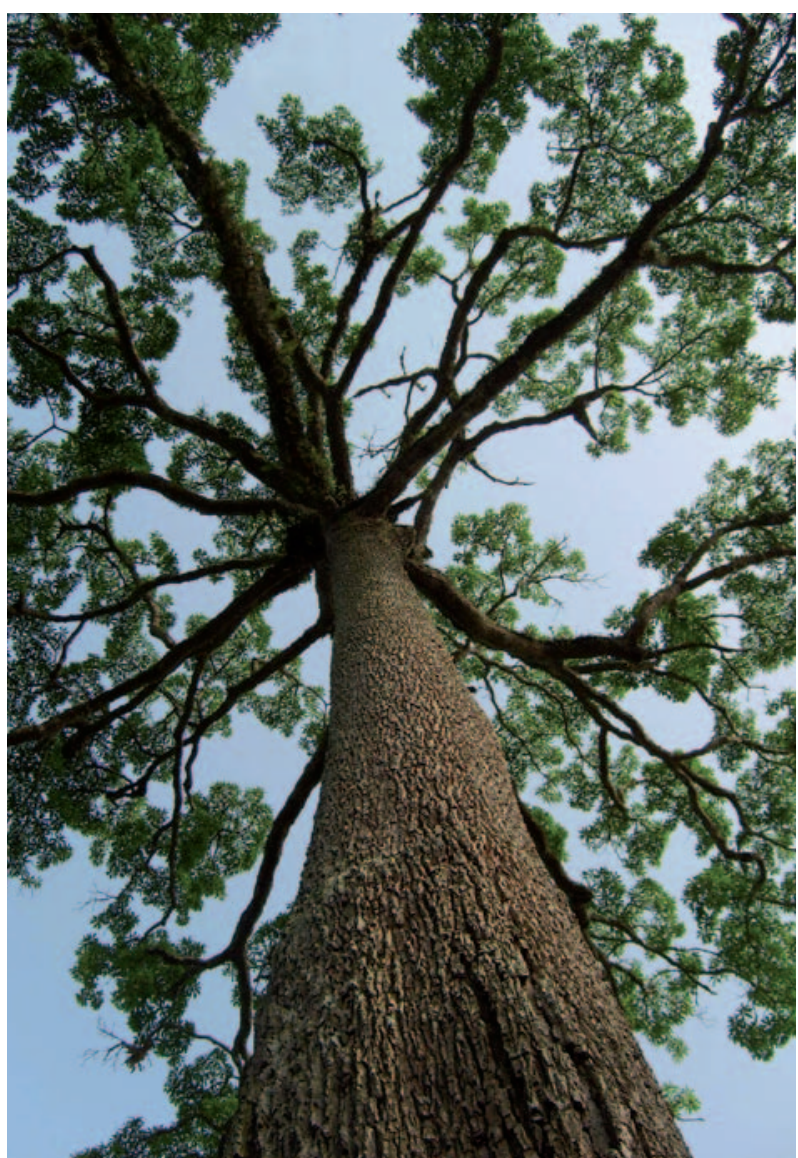

Photo 2.

Pied de moabi (Baillonella toxisperma).

Photo J.-L. Doucet.

l'Angola. Il est plus fréquent dans les forêts semi-caducifoliées que dans les forêts sempervirentes. C'est une espèce héliophile non pionnière et anémochore. Bien que tolérant d'importantes variations édaphiques, il préférerait les sols secs bien drainés de plateau ou de versant. Son accroissement en conditions naturelles varie entre 0,47 et $0,58 \mathrm{~cm} / \mathrm{an}$ en République centrafricaine, voire $0,64 \mathrm{~cm} / \mathrm{an}$ au Cameroun (Durrieu de Madron et al., 2000). La densité de son bois varie entre 0,5 et $0,63 \mathrm{~g} / \mathrm{cm}^{3}$ (Zanne et al., 2009).

\section{Le tali : Erythrophleum suaveolens (photo 4)}

L'espèce est distribuée du Sénégal au Soudan et au Kenya à l'Est, et au Zimbabawé et au Mozambique au Sud. Il est présent dans les forêts denses humides semi-caducifoliées et les galeries forestières (Duminil et al., 2010). Il est de tempérament héliophile pionnier (Hawthorne, 1995), principalement autochore et tolérant à des variations édaphiques importantes (Hall et al., 2004). L'accroissement moyen du diamètre en conditions naturelles est estimé à $0,45 \mathrm{~cm} /$ an en République centrafricaine et $0,68 \mathrm{~cm} / \mathrm{an}$ au Cameroun (Kouadio et al., 2014). La densité de son bois est comprise entre 0,75 et $0,98 \mathrm{~g} / \mathrm{cm}^{3}$ (Zanne et al., 2009).

\section{Collecte des données}

\section{Dispositif expérimental}

En 2009, un total de 387 placettes d'inventaire circulaires de $20 \mathrm{~m}$ de rayon (0,13 ha) a été installé autour de 136 assamela, 124 sapelli, 73 tali et 54 moabi. Ces arbres, distribués de manière relativement uniforme sur le site d'étude (figure 1), ont été sélectionnés en vue de couvrir une gamme aussi large que possible de diamètres (de 20 à $155 \mathrm{~cm}$ ) et d'intensités de compétition avec les arbres voisins (annexe 1). De ce fait, un total de $46 \%$ des arbres sélectionnés sont localisés en bordure d'ouvertures forestières datant de 5 à 10 ans (exploitation de 0,8 tige/ha) et $54 \%$ dans un environnement non perturbé par l'exploitation (figure 2).

\section{Suivi de la croissance}

Le diamètre $(D)$ de chaque arbre a été mesuré chaque année entre 2009 et 2012, à la même période (entre septembre et octobre). Le diamètre a été mesuré à un niveau de mesure identique marqué à la peinture (photo 5) situé à $1,30 \mathrm{~m}$ du sol ou à $30 \mathrm{~cm}$ au-dessus des contreforts le cas échéant (photo 6). L'accroissement annuel moyen périodique du diamètre ( $A M$ en $\mathrm{cm} / \mathrm{an}$ ) a été calculé pour chaque arbre sur l'ensemble de la période d'étude.

\section{Densité du peuplement et statut social des arbres}

Le diamètre $\left(D_{i}\right)$, ainsi que la distance à l'arbre focal (dist $t_{i}$ ) de tous les arbres compris dans les placettes circulaires et dont le diamètre était supérieur ou égal à $20 \mathrm{~cm}$ ont également été relevés en 2009. Ces données ont été utilisées pour calculer divers indices quantitatifs décrivant la densité du peuplement entourant l'arbre focal, le statut social et l'intensité de la compétition (tableau I). Le statut de la cime des arbres focaux par rapport à celle de leurs voisins a également été quantifié selon Doucet (2003) et Dawkins (1958). Tous ces indices sont indépendants des distances à l'exception de Gdist qui fait intervenir la distance entre l'arbre focal et ses voisins (tableau I).

\section{Conditions environnementales locales}

Un modèle numérique d'altitude avec une résolution spatiale de $30 \mathrm{~m}\left(\mathrm{SRTM}^{2}\right)$ a été utilisé pour déterminer l'altitude (Elev) à laquelle se trouve chaque arbre focal. Quatre variables hydrologiques susceptibles de traduire la teneur en eau du sol et donc d'influer sur la croissance des arbres ont également été calculées (tableau I) : l'indice de drainage (Flow accumulation ou Flacc), l'indice de position topographique (Topographic Position Index ou TPI) la surface drainée modifiée (Modified Catchment Area ou MCA) et l'indice d'humidité SAGA (SAGA Wetness Index ou $S W I$ ). Ces variables ont été dérivées du modèle numérique d'altitude à l'aide du logiciel SAGA-GIS (System for Automated Geoscientific Analyses 3 ).

2. https://lta.cr.usgs.gov/SRTM

3. https://www.saga-gis.org. 


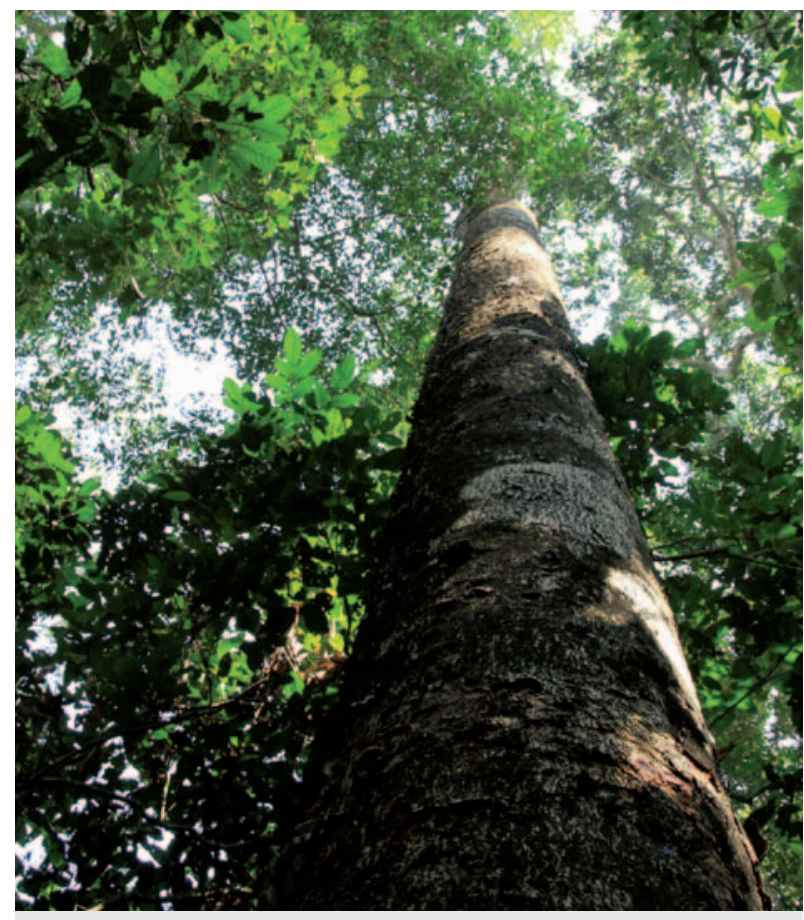

Photo 3.

Pied de sapelli (Entandrophragma cylindricum). Photo J.-L. Doucet.

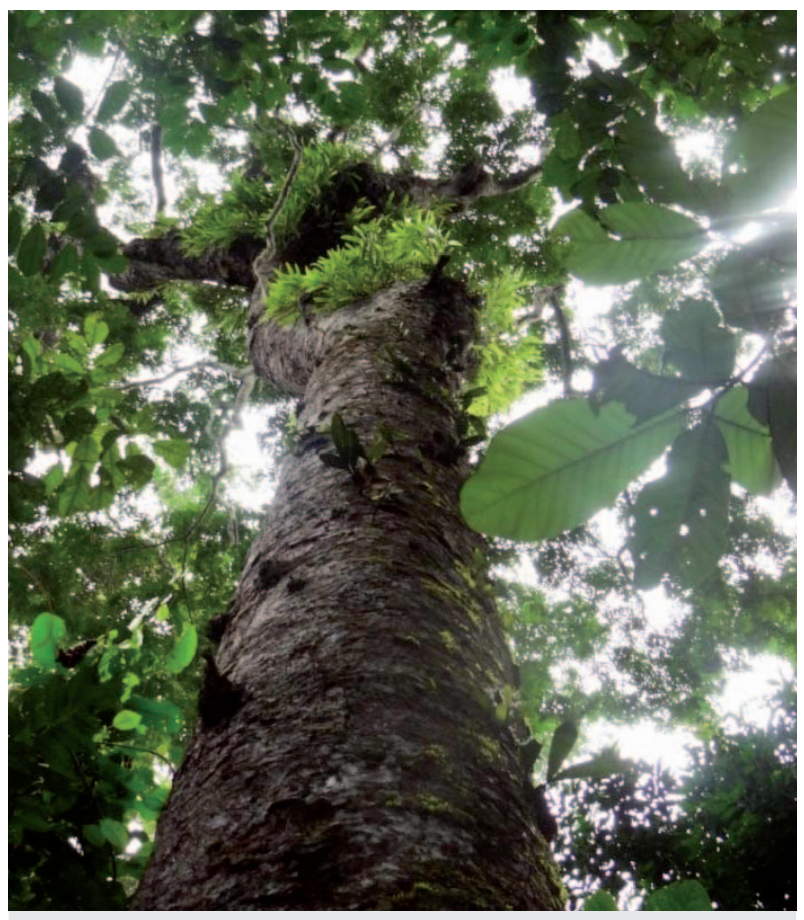

Photo 4.

Pied de tali (Erythrophleum suaveolens)

Photo F. Fétéké.
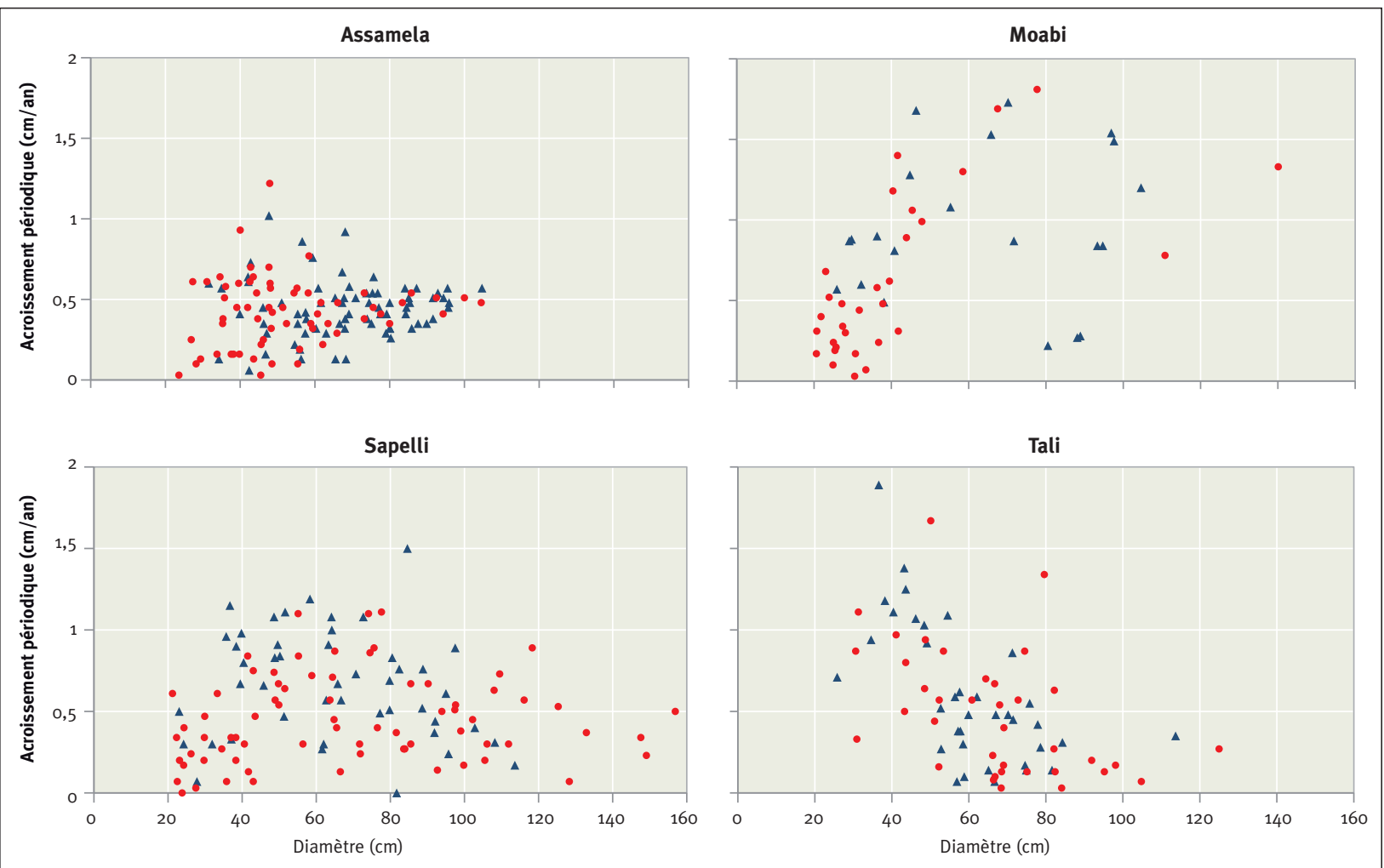

Figure 2.

Distribution de l'accroissement annuel moyen périodique du diamètre $(\mathrm{cm} / \mathrm{an})$ en fonction du diamètre initial (cm) pour les quatre essences étudiées. Pour couvrir la gamme de diamètres et d'intensités de la compétition, la croissance des arbres a été suivie dans un environnement perturbé $(\boldsymbol{\Lambda})$ ou non perturbé par l'exploitation forestière (๑). 


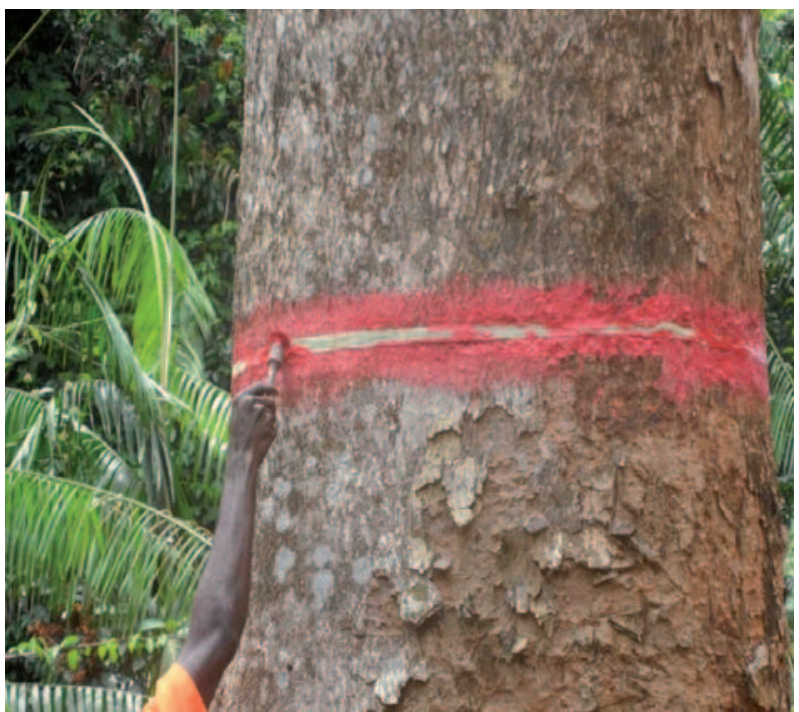

Photo 5.

Marquage du niveau de mesure du diamètre sur un pied de sapelli (Entandrophragma cylindricum).

Photo F. Fétéké.

\section{Analyses statistiques et modélisation}

Dans un premier temps, les accroissements annuels moyens périodiques mesurés ont été comparés aux valeurs de référence utilisées au Cameroun pour les quatre essences. Cette comparaison a été réalisée respectivement pour l'ensemble du jeu de données et séparément pour les arbres situés dans un environnement perturbé et non perturbé par l'exploitation forestière.

Ensuite, une série de modèles de croissance ont été ajustés aux données. La formulation générale de ces modèles traduit l'accroissement en fonction du diamètre et d'une variable exprimant le niveau de compétition et est dérivée de la fonction sigmoïde log-normale souvent employée pour ce type de modélisation (Obiang et al., 2012, 2013 ; Uriarte et al., 2004).

$$
A M_{e s t}=k_{1} \exp \left\{-\left[k_{2} \ln \left(\frac{k_{3}}{D}\right)\right]^{2}-k_{4} * X\right\}
$$

(équation 1)

$A M_{\text {est }}$ est l'accroissement annuel moyen périodique $k 1$, $k_{2}, k_{3}$ et $k_{4}$ sont les paramètres du modèle à ajuster, $D$ le diamètre des arbres et $X$ une variable de compétition ou environnementale, $k 1$ correspond à l'accroissement maximal (en $\mathrm{cm} / \mathrm{an}$ ) des arbres, $k 3$ le diamètre moyen (en $\mathrm{cm}$ ) des arbres présentant cet accroissement maximal, $k 2$ est un paramètre de forme de la courbe de croissance et $k 4$ est le paramètre qui dirige l'influence de la variable $X$.

Le modèle a tout d'abord été ajusté sans variable de compétition ou environnementale de manière à exprimer l'accroissement périodique en fonction du seul diamètre (modèle M1 ou modèle « diamétrique »). Différentes variables exprimant la densité locale et le statut social de l'arbre (tableau I) ont ensuite été comparées pour identifier celle qui permettait le mieux d'exprimer l'effet de la compétition sur la

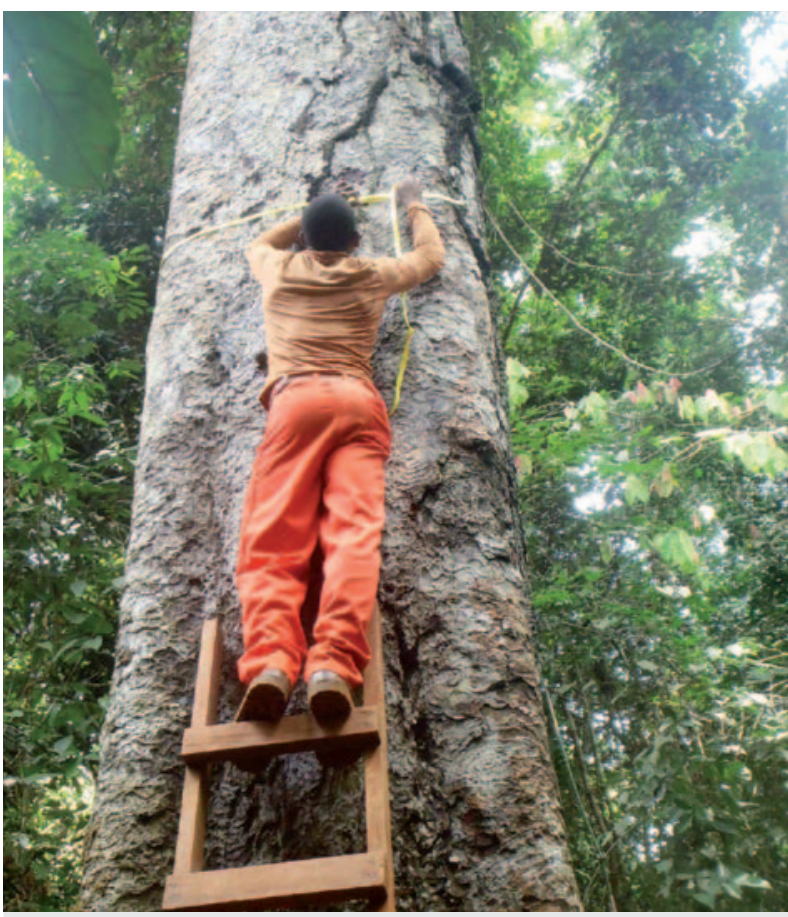

Photo 6.

Mesure du diamètre rehaussée au-dessus des contreforts sur un pied de tali (Erythrophleum suaveolens).

Photo C. Bouissou.

croissance en diamètre. Ces variables sont toutes construites de telle manière qu'une valeur nulle corresponde au niveau de pression minimal et que sa valeur soit directement proportionnelle à l'intensité de la compétition subie par l'arbre. Ces modèles de croissance peuvent ainsi être assimilés à des modèles de type accroissement potentiel $\times$ réducteur (modèles $\mathrm{M} 2$ ).

Les modèles ont été ajustés dans $R$ ( $R$ Development Core Team, 2013) à l'aide de la fonction gnls (Generalized Nonlinear Least Squares) fournie dans le package nlme (modèles linéaires ou non linéaires à effets mixtes (Pinheiro et al., 2013). Les performances des modèles ajustés ont été comparées grâce à différents critères statistiques : le critère d'information d'Akaike (AIC), l'erreur moyenne (ME), l'écart-type résiduel (ECTr) et le coefficient de détermination ajusté ( $\left.R^{2} a d j\right)$.

\section{Résultats}

\section{Variations de l'accroissement}

L'accroissement diamétrique varie en fonction de l'espèce et du diamètre des arbres au sein d'une même espèce (annexe 2). Il varie également selon le milieu. Les arbres situés en zone exploitée sont caractérisés par des accroissements supérieurs (de 5 à $50 \%$ ) à ceux des arbres situés dans des environnements non perturbés par l'exploitation (tableau II). Ces différences sont statistiquement 
Tableau I.

Indices décrivant l'intensité de la compétition et les conditions environnementales locales $D_{i}$ et $G_{i}$ sont le diamètre et la surface terrière respectivement de chaque arbre $i$ des $k$ arbres inventoriés dans la placette de surface $S$ et $k_{B}$ est le nombre d'arbres de diamètre plus grand que celui de l'arbre focal. Les indices topographiques et hydrologiques sont dérivés du modèle numérique d'élévation SRTM. $a$ est l'aire drainée par chaque pixel et $b$ est la pente locale.

\begin{tabular}{|c|c|c|}
\hline Indice & Définition & Réf. \\
\hline \multicolumn{3}{|c|}{ Intensité de la compétition } \\
\hline Nha & Nombre d'arbres voisins dans un rayon de $20 \mathrm{~m}$ autour de l'arbre focal (arbres/ha) : Nha $=\frac{k}{s}$ & (1) \\
\hline Gha & Somme des surfaces terrières des arbres voisins $\left(\mathrm{m}^{2} / \mathrm{ha}\right): G h a=\sum_{i=1}^{k} \frac{\pi}{4} D_{i}^{2}$ & (1) \\
\hline Gdist & $\begin{array}{l}\text { Somme des surfaces terrières des arbres voisins pondérées par la distance les séparant } \\
\text { de l'arbre focal (m/ha) : Gdist }=\sum_{i=1}^{k} \frac{G_{i}}{\text { dist }_{i}}\end{array}$ & (1) \\
\hline Nsup & Nombre d'arbres voisins de diamètre égal ou supérieur à l'arbre focal (arbres/ha) $: N s u p=\frac{k_{B}}{s}$ & (1) \\
\hline Gsup & $\begin{array}{l}\text { Somme des surfaces terrières des arbres voisins de diamètre égal ou supérieur à l'arbre focal (m²/ha): } \\
\text { Gsup }=\sum_{i=1}^{k B} \frac{\pi}{4} D_{i}^{2}\end{array}$ & (1) \\
\hline Nrel & Densité relative des arbres voisins de diamètre égal ou supérieur à celui de l'arbre focal (ratio) $: N r e l=\frac{k_{B}}{k}$ & - \\
\hline Grel & $\begin{array}{l}\text { Surface terrière relative des arbres voisins de diamètre égal ou supérieur à celui de l'arbre focal (ratio): } \\
\text { Grel }=\frac{\sum_{i=1}^{k_{B}} \frac{\pi}{4} D_{i}^{2}}{\frac{\pi}{4} D_{i}^{2}}\end{array}$ & - \\
\hline Sc & $\begin{array}{l}\text { Position de la cime de l'arbre dans la canopée : } \\
2=S c_{1}: \text { cime dominée dépassée par une ou plusieurs autres cimes voisines } \\
1=S C 2: \text { cime voisine d'une ou de plusieurs autres cimes sans pour autant être dominée par celle(s)-ci } \\
0=S c 3: \text { cime au-dessus de toutes les autres cimes voisines }\end{array}$ & (2) \\
\hline Daw & $\begin{array}{l}\text { Exposition de la cime à la lumière: } \\
4=\text { Daw } 1: \text { cime non exposée d'arbre de la canopée inférieure } \\
3=\text { Daw } 2: \text { cime peu exposée d'arbre de la canopée inférieure } \\
2=\text { Daw } 3: \text { cime modérément exposée d'arbre de la canopée supérieure } \\
1=\text { Daw } 4: \text { cime exposée d'arbre de la canopée supérieure } \\
0=\text { Daw } 5: \text { cime fortement exposée d'arbre émergent }\end{array}$ & (3) \\
\hline \multicolumn{3}{|c|}{ Conditions environnementales locales } \\
\hline Elev & Altitude locale liée à la position des arbres (m) & - \\
\hline Flacc & $\begin{array}{l}\text { «Flow Accumulation Index » obtenu par évaluation de l'aire de la zone de décharge en amont de chaque } \\
\text { cellule de la grille d'analyse }\left(\mathrm{m}^{2}\right)\end{array}$ & (4) \\
\hline TPI & $\begin{array}{l}\text { Topographic Position Index (-) : indice d'humidité mesurant la position topographique relative du pixel } \\
\text { central par rapport à celles des pixels dans un rayon d'un kilomètre autour et donnant une idée du temps } \\
\text { plus ou moins long de rétention d'eau sur chaque pixel. } \\
\text { Plus l'indice est élevé, plus l'aire amont contributive est grande et la pente faible, l'eau a donc tendance } \\
\text { à stagner : TPI = In }[a / \tan (b)]\end{array}$ & (5) \\
\hline$M C A$ & $\begin{array}{l}\text { Modified Catchment Area }\left(\mathrm{m}^{2}\right) \text { : indice modifié d'humidité calculé sur la base d'une valeur modifiée } \\
\text { de l'aire de drainage de façon à donner une estimation plus réaliste de l'humidité du sol }\end{array}$ & (5) \\
\hline SWI & $\begin{array}{l}\text { Saga Wetness Index (-) : indice prédisant l'humidité du sol en considérant le rapport entre le logarithme } \\
\text { de } M C A \text { et la tangente de la pente locale }: S W I=\ln [M C A / \tan (b)]\end{array}$ & (5) \\
\hline
\end{tabular}

Remarque : Les références indiquées dans le tableau correspondent respectivement à : (1) Steneker et Jarvis, 1963 ; (2) Doucet, 2003 ; (3) Dawkins, 1958 ; (4) Beven et Kirkby, 1979 ; (5) Boehner et al., 2002. 
Tableau II.

Comparaison des valeurs d'accroissement de référence $\left(A M_{r e p} \mathrm{~cm} / \mathrm{an}\right)$ utilisées par l'administration forestière du Cameroun aux accroissements périodiques moyens $(A M, \mathrm{~cm} / \mathrm{an})$ par essence observés sur des arbres situés en milieu perturbé ou non par l'exploitation forestière. L'intensité de l'exploitation ainsi que l'âge des perturbations sont indiqués. Les valeurs indiquées en italique correspondent aux intervalles de confiance (1- $\alpha=95 \%)$. Le nombre d'arbres $(N)$ mesurés est indiqué par essence et selon le milieu.

\begin{tabular}{|c|c|c|c|c|c|c|c|c|c|c|c|}
\hline \multirow{3}{*}{ Essence } & \multirow{3}{*}{$A M_{\text {ref }}$} & \multicolumn{2}{|c|}{$\begin{array}{l}\text { Milieu non } \\
\text { exploité }\end{array}$} & \multicolumn{6}{|c|}{ Milieu exploité (prélèvement de 0,8 tige/ha) } & \multirow{2}{*}{\multicolumn{2}{|c|}{ Total }} \\
\hline & & & & \multicolumn{2}{|c|}{ Moins de 5 ans } & \multicolumn{2}{|c|}{ 5-10 ans } & \multicolumn{2}{|c|}{ Moyenne } & & \\
\hline & & $N$ & $A M$ & $N$ & $A M$ & $N$ & $A M$ & $N$ & $A M$ & $N$ & $A M$ \\
\hline $\begin{array}{l}\text { Assamela } \\
\text { Pericopsis elata }\end{array}$ & 0,4 & 63 & $0,42 \pm 0,05$ & 26 & $0,42 \pm 0,08$ & 47 & $0,46 \pm 0,05$ & 73 & $0,45 \pm 0,04$ & 136 & $0,43 \pm 0,03$ \\
\hline $\begin{array}{l}\text { Moabi } \\
\text { Baillonella } \\
\text { toxisperma }\end{array}$ & 0,4 & 33 & $0,58 \pm 0,20$ & 1 & $0,49 \pm 0,00$ & 20 & $0,97 \pm 0,20$ & 21 & $0,88 \pm 0,18$ & 54 & $0,73 \pm 0,14$ \\
\hline $\begin{array}{l}\text { Sapelli } \\
\text { Entandrophragma } \\
\text { cylindricum }\end{array}$ & 0,5 & 77 & $0,42 \pm 0,06$ & 37 & $0,68 \pm 0,11$ & 10 & $0,57 \pm 0,23$ & 47 & $0,65 \pm 0,10$ & 124 & $0,51 \pm 0,06$ \\
\hline $\begin{array}{l}\text { Tali } \\
\text { Erythrophleum } \\
\text { suaveolens }\end{array}$ & 0,4 & 37 & $0,49 \pm 0,13$ & 21 & $0,62 \pm 0,2$ & 15 & $0,58 \pm 0,19$ & 36 & $0,60 \pm 0,14$ & 73 & $0,55 \pm 0,10$ \\
\hline
\end{tabular}

significatives pour le moabi et le sapelli. On constate également que la valeur de référence préconisée par l'administration forestière au Cameroun pour le sapelli est significativement supérieure de $20 \%$ à l'accroissement mesuré en milieu non perturbé. Pour les trois autres essences, il n'existe pas de différence significative entre les accroissements de référence et les valeurs moyennes observées en milieu non perturbé.

\section{Modèles à une seule variable explicative (M1)}

Le modèle diamétrique $M_{1}$ a été calibré en ajustant, pour chaque essence, une valeur unique des paramètres $k 1$, $k_{2}$ et $k_{3}$ (modèle M1a ; tableau III). Cette manière de procéder n'a pas permis d'obtenir une estimation significativement différente de 0 pour les paramètres $k_{2}$ et $k_{3}$, probablement en raison de l'importante corrélation existant entre ces deux paramètres. Une calibration satisfaisante du modèle a été obtenue en fixant une valeur commune du paramètre de forme $k 2$ pour les quatre essences (modèle M1b ; tableau III). Cette modification n'altère pas les performances du modèle.

Le modèle $M_{1} b$ permet d'expliquer le tiers de la variabilité de l'accroissement courant en diamètre toutes essences confondues. La part de variance expliquée est néanmoins fortement variable en fonction de l'essence considérée. Le meilleur ajustement est obtenu pour le moabi $\left(R^{2}=42 \%\right)$, suivi par le tali (36\%), le sapelli (24\%) et enfin par l'assamela $(-6,4 \%)$. Le coefficient de détermination négatif obtenu pour l'assamela signifie que le modèle ajusté est moins performant qu'une simple moyenne.
Le moabi est l'essence qui présente la vitesse de croissance la plus élevée et la mieux soutenue, elle atteint un maximum de $1,2 \mathrm{~cm} /$ an à $70 \mathrm{~cm}$ de diamètre (figure 3). Le sapelli est caractérisé par une vitesse de croissance relativement faible mais également bien soutenue qui atteint son maximum $(0,7 \mathrm{~cm} / \mathrm{an})$ vers $60 \mathrm{~cm}$ de diamètre. Le tali se distingue des autres essences par une croissance juvénile rapide atteignant $1 \mathrm{~cm} /$ an vers $30 \mathrm{~cm}$ de diamètre mais qui diminue ensuite très rapidement.

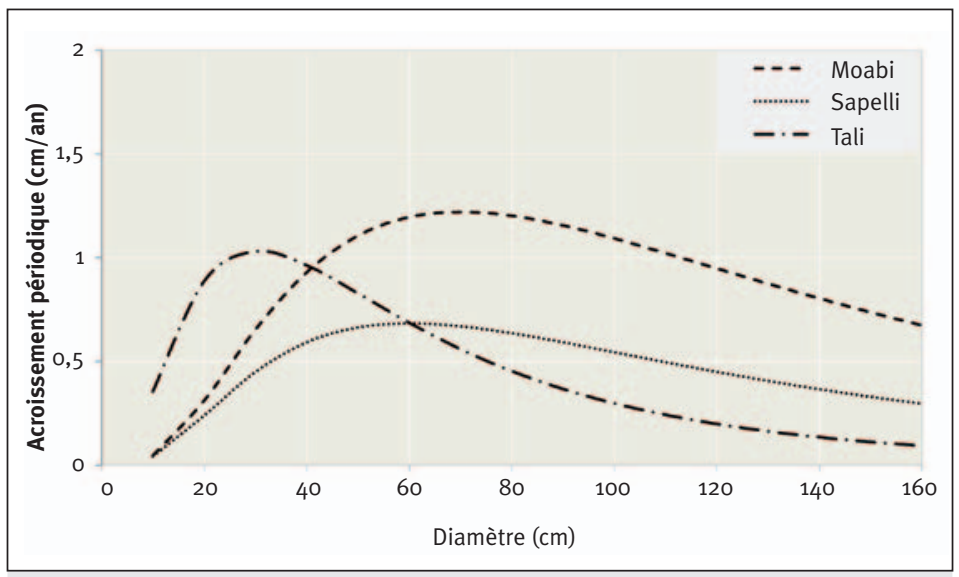

Figure 3.

Représentation graphique du modèle $M 1 b$ reliant l'accroissement annuel moyen périodique (en $\mathrm{cm} / \mathrm{an}$ ) au diamètre (en $\mathrm{cm}$ ) pour les trois essences étudiées (moabi, Baillonella toxisperma, sapelli, Entandrophragma cylindricum, tali, Erythrophleum suaveolens) pour lesquelles le modèle est significativement plus performant qu'une simple moyenne. 
Tableau III.

Ajustement des modèles diamétriques $M_{1}$ et $M_{1} b$ et du modèle $M_{2}$ basé sur la variable Gsup pour les quatre essences étudiées. Les valeurs estimées des paramètres sont données pour chaque essence, les valeurs indiquées en italique correspondent aux intervalles de confiance $(1-\alpha=95 \%)$. Les critères statistiques de qualité de l'ajustement du modèle sont donnés pour chaque essence et pour le modèle dans son ensemble.

\begin{tabular}{|c|c|c|c|c|c|c|}
\hline Essence & k1 & $k 2$ & $k 3$ & $k 4$ & ECTr & $R^{2}$ adj \\
\hline \multicolumn{7}{|l|}{ Modèle M1a } \\
\hline $\begin{array}{l}\text { Assamela } \\
\text { Pericopsis elata }\end{array}$ & $0,45 \pm 0,06$ & $0,48 \pm 0,98$ & $75,3 \pm 106,8$ & & 0,197 & 0,001 \\
\hline $\begin{array}{l}\text { Moabi } \\
\text { Baillonella toxisperma }\end{array}$ & $1,22 \pm 0,17$ & $1,16 \pm 1,01$ & $69,9 \pm 107,2$ & & 0,397 & 0,415 \\
\hline $\begin{array}{l}\text { Sapelli } \\
\text { Entandrophragma cylindricum }\end{array}$ & $0,70 \pm 0,11$ & $1,21 \pm 1,01$ & $59,7 \pm 107,0$ & & 0,288 & 0,240 \\
\hline $\begin{array}{l}\text { Tali } \\
\text { Erythrophleum suaveolens }\end{array}$ & $1,02 \pm 0,17$ & $1,49 \pm 1,07$ & $35,4 \pm 107,0$ & & 0,330 & 0,366 \\
\hline Total & & & & & 0,287 & 0,347 \\
\hline \multicolumn{7}{|l|}{ Modèle M1b } \\
\hline $\begin{array}{l}\text { Assamela } \\
\text { Pericopsis elata }\end{array}$ & $0,49 \pm 0,06$ & \multirow{4}{*}{$1,15 \pm 0,16$} & $59,1 \pm 9,0$ & & 0,203 & $-0,064$ \\
\hline $\begin{array}{l}\text { Moabi } \\
\text { Baillonella toxisperma }\end{array}$ & $1,22 \pm 0,14$ & & $70,0 \pm 11,4$ & & 0,394 & 0,423 \\
\hline $\begin{array}{l}\text { Sapelli } \\
\text { Entandrophragma cylindricum }\end{array}$ & $0,68 \pm 0,09$ & & $60,0 \pm 10,8$ & & 0,287 & 0,243 \\
\hline $\begin{array}{l}\text { Tali } \\
\text { Erythrophleum suaveolens }\end{array}$ & $1,03 \pm 0,22$ & & $30,3 \pm 10,7$ & & 0,331 & 0,362 \\
\hline Total & & & & & 0,288 & 0,341 \\
\hline \multicolumn{7}{|l|}{ Modèle M2 } \\
\hline $\begin{array}{l}\text { Assamela } \\
\text { Pericopsis elata }\end{array}$ & $0,67 \pm 0,17$ & \multirow{4}{*}{$1,10 \pm 0,15$} & $46,4 \pm 9,6$ & $0,032 \pm 0,021$ & 0,188 & 0,092 \\
\hline $\begin{array}{l}\text { Moabi } \\
\text { Baillonella toxisperma }\end{array}$ & $1,40 \pm 0,26$ & & $61,2 \pm 12,5$ & $0,031 \pm 0,028$ & 0,378 & 0,470 \\
\hline $\begin{array}{l}\text { Sapelli } \\
\text { Entandrophragma cylindricum }\end{array}$ & $0,78 \pm 0,21$ & & $55,0 \pm 11,5$ & $0,018 \pm 0,025$ & 0,283 & 0,268 \\
\hline $\begin{array}{l}\text { Tali } \\
\text { Erythrophleum suaveolens }\end{array}$ & $1,77 \pm 0,67$ & & $24,5 \pm 10,1$ & $0,031 \pm 0,026$ & 0,308 & 0,449 \\
\hline Total & & & & & 0,275 & 0,402 \\
\hline
\end{tabular}

\section{Modèles incluant l'intensité de la compétition (M2)}

De nouveaux ajustements ont ensuite été réalisés pour ajouter au modèle diamétrique (M1b) une variable exprimant l'intensité de la compétition. Les sept variables exprimant la compétition ont été comparées pour identifier celle(s) qui expliquai(en)t le mieux la part de variabilité résiduelle du modèle $M_{1} b$ (annexe 3 ). Les modèles faisant intervenir la variable Nsup étaient les plus performants en termes de $R^{2}$, mais convergeaient difficilement et conduisaient à des valeurs de paramètres aberrantes au plan de leur interprétation biologique. Ils ont donc été écartés au profit d'un modèle basé sur la variable Gsup qui fournit de bons ajustements (tableau III).

Le modèle obtenu peut être assimilé à un modèle de type potentiel $x$ réducteur où l'accroissement potentiel est fonction de l'essence et du diamètre, la fonction de réduction étant exprimée par la surface terrière des arbres voisins 
de diamètre supérieur (Gsup). L'influence de la variable Gsup sur l'accroissement est significative pour l'assamela, le moabi et le tali. La diminution de l'écart-type résiduel suite à l'introduction de la variable dans le modèle peut sembler relativement faible (environ $5 \%$ ) mais son impact sur l'accroissement n'est absolument pas négligeable (figure 4). Les valeurs ajustées des paramètres $k_{3}$ du modèle $M_{2}$ (tableau III) sont systématiquement inférieures à celles qui avaient été estimées lors du calibrage du modèle Mib. Cela indique que l'accroissement périodique moyen maximal est généralement atteint plus tard que l'accroissement potentiel maximal. Cela est à mettre en parallèle avec le fait que la variable est négativement corrélée au diamètre de l'arbre focal ( $R=-0,695)$, indiquant que les arbres plus petits subissent en moyenne une pression de compétition supérieure aux arbres plus gros.

\section{Influence des conditions environnementales locales}

L'influence des cinq variables topographiques et hydrologiques sur l'accroissement des arbres focaux a ensuite été analysée. Aucune corrélation linéaire significative n'est observée entre ces variables et les résidus du modèle $M_{2}$ (tableau IV). Des analyses complémentaires (analyses graphiques et tests de régression polynomiale) n'ont pas permis de détecter de relation entre ces variables environnementales locales et la croissance des arbres étudiés.

\section{Discussion}

Le but était ici d'étudier les variations de la croissance diamétrique pour quatre essences commerciales largement exploitées dans les forêts semi-décidues du Sud-Est du Cameroun et de développer un modèle de croissance intégrant le diamètre, l'intensité de la compétition et les conditions environnementales locales pour ces essences. L'accroissement observé sur un jeu de 387 arbres répartis sur une large gamme de diamètres et suivis pendant trois ans a permis de mettre en évidence des différences significatives de croissance en fonction de l'ouverture de la canopée, entre milieu perturbé et non perturbé par l'exploitation forestière. Ces résultats confirment l'importance de la lumière pour la croissance des arbres tropicaux (e.g. Baker et al., 2003) et l'effet « stimulant » de l'exploitation sur la dynamique forestière (Gourlet-Fleury et al., 2013). La croissance observée en milieu non perturbé ne présente pas de différence significative avec les valeurs de référence préconisées par l'administration forestière du Cameroun pour trois des quatre essences étudiées (assamela, moabi et tali). Pour le sapelli, la valeur de référence de l'administration est de $20 \%$ supérieure à la valeur moyenne observée, ce qui indique une surestimation des taux de reconstitution de cette essence dans les plans d'aménagement établis à partir de cette valeur. En outre, la croissance observée en milieu perturbé est de $10 \%$ (cas de l'assamela) à $120 \%$ (cas du moabi) supérieure à la valeur de référence de l'adminis-
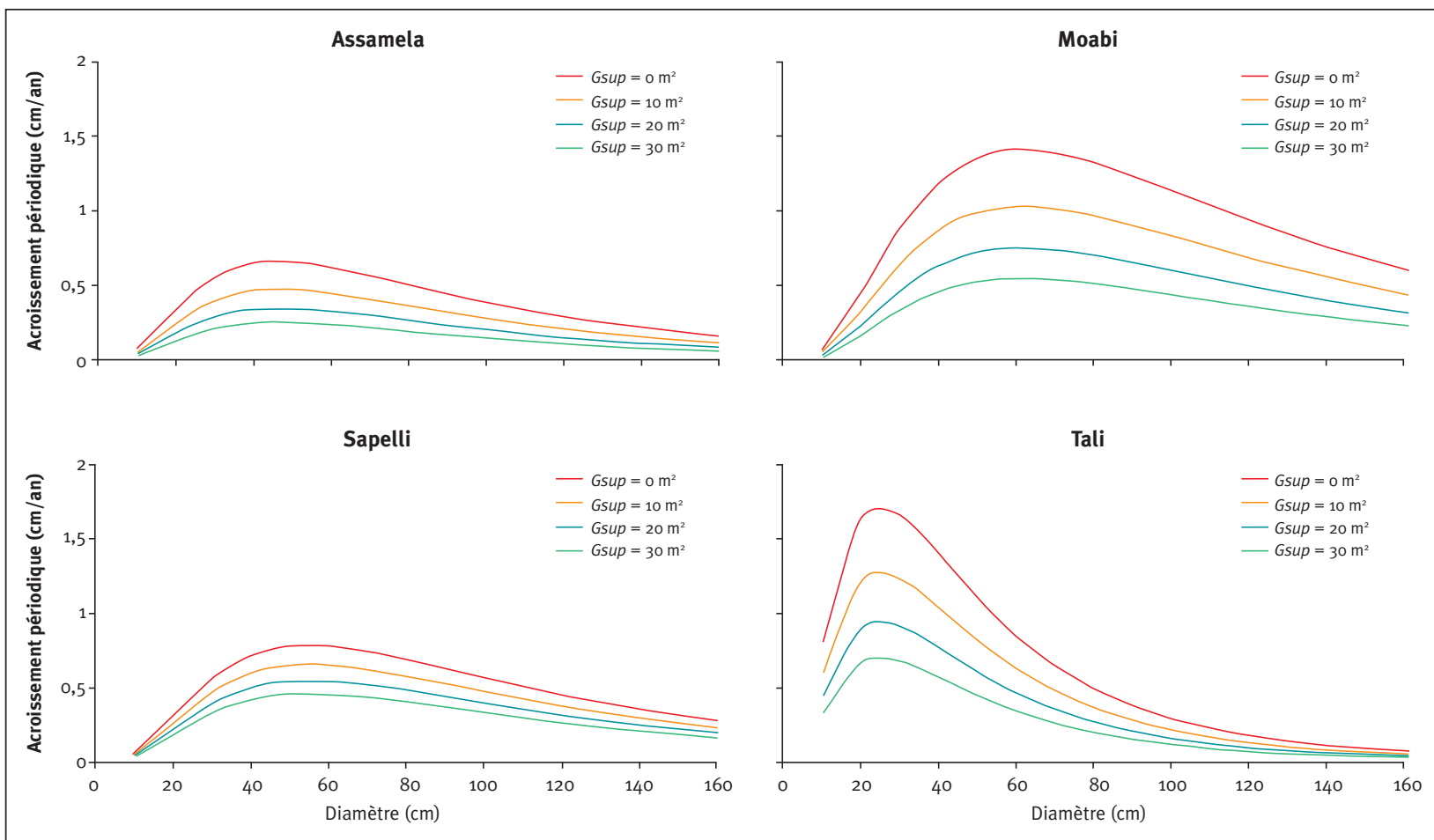

Figure 4.

Représentation graphique du modèle M2 reliant l'accroissement annuel moyen périodique (en cm/an) au diamètre (en cm) et à la surface terrière des arbres plus gros que l'arbre focal (Gsup, en $\mathrm{m}^{2} / \mathrm{ha}$ ) pour les quatre essences étudiées. 
Tableau IV.

Corrélation linéaire entre les résidus de l'ajustement du modèle

M2 et les cinq variables topographiques et hydrologiques :

l'altitude (Elev), l'indice de drainage (Flow accumulation ou Flacc), l'indice de position topographique (Topographic Position Index ou TPI), la surface drainée modifiée (Modified Catchment Area ou $M C A)$ et l'indice d'humidité SAGA (SAGA Wetness Index ou SWI).

\begin{tabular}{|l|c|c|c|c|c|}
\hline Essence & Elev & Flacc & TPI & MCA & SWI \\
\hline $\begin{array}{l}\text { Assamela } \\
\text { Pericopsis elata }\end{array}$ & $-0,06$ & 0,04 & 0,03 & $-0,02$ & 0,16 \\
\hline $\begin{array}{l}\text { Moabi } \\
\text { Baillonella } \\
\text { toxisperma }\end{array}$ & 0,06 & $-0,21$ & 0,14 & 0,12 & 0,00 \\
\hline $\begin{array}{l}\text { Sapelli } \\
\text { Entandrophragma } \\
\text { cylindricum }\end{array}$ & $-0,07$ & 0,00 & 0,04 & 0,07 & $-0,03$ \\
\hline $\begin{array}{l}\text { Tali } \\
\text { Erythrophleum } \\
\text { suaveolens }\end{array}$ & 0,21 & $-0,04$ & 0,05 & 0,00 & 0,02 \\
\hline
\end{tabular}

tration, et pourrait avoir comme conséquence une sousestimation des taux de reconstitution de ces essences en seconde rotation. La révision des plans d'aménagement qui s'amorce au Cameroun devra donc, pour ces essences, prendre en compte une valeur de croissance révisée en plus des tarifs de cubage (Fayolle et al., 2013). Les valeurs moyennes d'accroissement observées dans le cadre de cette étude se rapprochent des résultats d'autres études antérieures sur les mêmes essences (Bourland et al., 2012 ; Kouadio et al., 2014 ; Durrieu de Madron et al., 2000).

Pour trois des quatre essences étudiées, une proportion relativement importante des variations de l'accroissement annuel moyen est expliquée par le modèle log-normal dépendant uniquement du diamètre $\left(R^{2}>0,24\right.$ pour le moabi, le sapelli et le tali). Ce type de courbe de croissance unimodale en fonction du diamètre a largement été rapporté pour les arbres tropicaux (e. g. Hérault et al., 2011 ; Obiang et al., 2012, 2013). L'hypothèse pour expliquer ce patron repose sur le fait que la taille des arbres suit généralement une courbe sigmoïde en fonction du temps, et qu'après une période initiale d'augmentation de la croissance le taux de croissance diminue (Weiner et Thomas, 2001). Si la forme de la courbe est générale, l'amplitude des variations de croissance au cours de la vie de l'arbre diffère fortement entre les essences d'un même site et la croissance maximale est généralement observée à $60 \%$ de la taille maximale (Hérault et al., 2011). Dans le cadre de cette étude, les variations de croissance sont extrêmement importantes pour le tali et le moabi, qui atteignent jusque 1 et $1,2 \mathrm{~cm} / \mathrm{an}$ respectivement à 30 et $70 \mathrm{~cm}$ de diamètre. Ces variations sont moindres pour l'assamela et le sapelli. D'ailleurs, dans le cas de l'assamela, le modèle log-normal est non significatif et un accroissement moyen devrait lui être préféré. L'assamela est l'essence pour laquelle la variabilité des accroissements observés est la plus faible avec un coefficient de variation de $47 \%$, contre des valeurs allant de $65 \%$ à $75 \%$ pour les trois autres essences.

Si le modèle diamétrique s'ajuste bien aux données pour trois des quatre essences étudiées, le modèle $M 2$, qui prend en compte l'intensité de la compétition, améliore la prédiction de l'accroissement diamétrique dans une proportion d'environ $10 \%$ pour le moabi et le sapelli et de près de $25 \%$ pour le tali. Le modèle $M_{2}$ est significatif pour l'assamela, mais son coefficient de détermination reste malgré tout très faible $(<10 \%)$. La variable Gsup retenue pour exprimer l'intensité de la compétition est fréquemment utilisée dans les modèles de croissance dévolus aux peuplements de structure irrégulière (Lejeune, 1996 ; Ledermann et Eckmüllner, 2004 ; Zhao et al., 2004). D'autres auteurs retiennent également des variables plus simples de la structure des peuplements telles que la surface terrière pour l'okoumé (Obiang et al., 2013) et/ou la densité du peuplement pour l'azobé au Gabon (Obiang et al., 2012). Les gains de précision obtenus à partir du modèle $M_{2}$ montrent bien l'intérêt de prendre en compte l'influence du peuplement local dans l'aménagement forestier (Therrell et al., 2007). Les variables explicatives ( $D h p$ et Gsup) peuvent être dérivées de données issues des inventaires forestiers tels que réalisés actuellement en Afrique centrale, ce qui permet l'utilisation de ce modèle pour prédire des valeurs de croissance plus précises que celles préconisées par l'administration pour ces essences. Toutefois, les gains de précision à espérer en utilisant le modèle $M_{2}$ seront sans doute inférieurs à ceux présentés ci-avant dans la mesure où la variable Gsup est calculée pour chaque arbre au sein de parcelles d'inventaire de forme généralement allongée (typiquement $250 \mathrm{~m} \times 20 \mathrm{~m}$ ), alors que les arbres utilisés pour construire le modèle $M 2$ sont toujours situés au centre d'une placette circulaire de 13 ares, qui permet de mieux rendre compte de la compétition s'exerçant sur l'arbre focal. Dans le cas où les données d'inventaire ne sont pas issues de parcelles d'échantillonnage de surface connue (cas d'un inventaire de prospection, par exemple), une détermination du statut social (selon le protocole de Doucet, 2003) pourrait néanmoins permettre une approximation de Gsup et l'utilisation du modèle $M_{2}$ (tableau V). Le modèle se présente toutefois comme une sous-composante du modèle matriciel simplifié habituellement utilisé en Afrique centrale dans le calcul des taux de reconstitution des stocks exploitables (Picard et al., 2008) ou d'autre modèles plus élaborés à envisager pour simuler la dynamique globale des populations d'essences exploitées.

Les caractéristiques topographiques et hydrologiques considérées dans l'analyse ne permettent pas d'améliorer les performances prédictives du modèle $M_{2}$. Ce résultat peut s'expliquer en partie par le fait que l'alimentation en eau ne constitue sans doute pas un facteur limitant pour les essences étudiées dans la zone d'étude. Une autre explication concerne la précision relativement grossière des données de base utilisées pour calculer ces variables. Le SRTM est un modèle numérique de surface et non un modèle numérique de terrain. Il combine, avec une résolution spatiale de $30 \mathrm{~m}$, les variations d'altitude et de hauteur du couvert végétal (Reuter et al., 2007). 
Tableau V.

Valeur moyenne de Gsup correspondant au statut de l'arbre. Les valeurs indiquées en italique correspondent aux intervalles de confiance (1- $\alpha=95 \%)$.

Toutes les valeurs sont exprimées en $\mathrm{m}^{2}$.

\begin{tabular}{|l|c|c|c|}
\hline \multirow{2}{*}{ Essence } & \multicolumn{3}{|c|}{ Statut social } \\
\cline { 2 - 4 } & Dominant & $\begin{array}{c}\text { Co- } \\
\text { dominant }\end{array}$ & Dominé \\
\hline $\begin{array}{l}\text { Assamela } \\
\text { Pericopsis elata }\end{array}$ & $5,3 \pm 2,97$ & $7,1 \pm 1,34$ & $15,8 \pm 3,18$ \\
\hline $\begin{array}{l}\text { Moabi } \\
\text { Baillonella } \\
\text { toxisperma }\end{array}$ & $4,3 \pm 2,46$ & $4 \pm 2,64$ & $16,9 \pm 2,64$ \\
\hline $\begin{array}{l}\text { Sapelli } \\
\text { Entandrophragma } \\
\text { cylindricum }\end{array}$ & $4,1 \pm 1,59$ & $4,8 \pm 1,99$ & $19,2 \pm 3,03$ \\
\hline $\begin{array}{l}\text { Tali } \\
\text { Erythrophleum } \\
\text { suaveolens }\end{array}$ & $3,4 \pm 3,08$ & $5,4 \pm 2,48$ & $10,5 \pm 2,61$ \\
\hline \begin{tabular}{l} 
Total \\
\hline
\end{tabular} & $4,3 \pm 1,16$ & $6,1 \pm 0,98$ & $15,7 \pm 1,55$ \\
\hline
\end{tabular}

\section{Conclusion}

Dans cette étude, deux modèles de croissance dépendant du diamètre $\left(M_{1} b\right)$ et de l'intensité de la compétition (M2) ont pu être établis pour quatre essences commerciales extrêmement importantes dans le Sud-Est du Cameroun : l'assamela, Pericopsis elata (Fabaceae), le moabi, Baillonella toxisperma (Sapotaceae), le sapelli, Entandrophragma cylindricum (Meliaceae), et le tali, Erythrophleum suaveolens (Fabaceae, Caesalpioniodeae). Sur le plan pratique, la mise en œuvre de ces modèles est compatible avec des données d'inventaire d'aménagement récoltées au sein de parcelles d'échantillonnage de surface connue. Le modèle $M 2$ nécessite un traitement préalable des données un peu plus élaboré (calcul de la variable ), mais facilement intégrable dans une procédure informatisée de traitement de données d'inventaire.

L'intégration des modèles présentés dans cette étude dans une plateforme de simulation telle que Capsis (DufourKowalski et al., 2012) constitue une suite logique en vue d'une utilisation opérationnelle de ces modèles par les aménagistes forestiers. Cette démarche est en cours et permettra de comparer des simulations basées sur différentes approches (DME et taux de reconstitution actuels en vigueur et description de la croissance à l'aide des nouveaux modèles).

Annexe 1.

Statistiques descriptives unidimensionnelles (minimum, maximum, moyenne et écart-type) par essence des différentes variables du jeu de données décrivant le diamètre des arbres focaux, l'intensité de la compétition et les conditions environnementales locales.

\begin{tabular}{|c|c|c|c|c|c|c|c|c|c|c|c|c|c|c|c|c|}
\hline \multirow[t]{2}{*}{ Variable } & \multicolumn{4}{|c|}{$\begin{array}{c}\text { Assamela } \\
\text { Pericopsis elata }\end{array}$} & \multicolumn{4}{|c|}{$\begin{array}{c}\text { Moabi } \\
\text { Baillonella toxisperma }\end{array}$} & \multicolumn{4}{|c|}{$\begin{array}{c}\text { Sapelli } \\
\text { Entandrophragma cylindricum }\end{array}$} & \multicolumn{4}{|c|}{$\begin{array}{l}\text { Tali } \\
\text { Erythrophleum suaveolens }\end{array}$} \\
\hline & MIN & MAX & MOY & ECT & MIN & MAX & MOY & ECT & MIN & MAX & MOY & ECT & MIN & MAX & MOY & ECT \\
\hline$D(\mathrm{~cm})$ & 23,60 & 104,70 & 61,04 & 19,47 & 20,60 & 140,10 & 49,33 & 28,51 & 21,00 & 156,90 & 65,75 & 31,65 & 25,80 & 125,00 & 63,34 & 19,35 \\
\hline$A M(\mathrm{~cm} / \mathrm{an})$ & 0,03 & 1,22 & 0,43 & 0,20 & $-0,07$ & 1,81 & 0,73 & 0,52 & $-0,10$ & 1,50 & 0,51 & 0,33 & 0,03 & 1,89 & 0,55 & 0,41 \\
\hline Nha (ha) & 31,85 & 294,59 & 155,84 & 57,48 & 15,92 & 270,70 & 121,49 & 50,38 & 15,92 & 278,66 & 149,73 & 55,94 & 15,92 & 278,66 & 133,71 & 58,84 \\
\hline Nsup (ha) & 0,00 & 175,16 & 29,45 & 34,96 & 0,00 & 167,20 & 49,98 & 46,26 & 0,00 & 230,89 & 31,59 & 48,55 & 0,00 & 103,50 & 17,34 & 22,45 \\
\hline Nrel & 0,00 & 1,00 & 0,20 & 0,21 & 0,00 & 1,00 & 0,41 & 0,34 & 0,00 & 0,96 & 0,19 & 0,26 & 0,00 & 0,68 & 0,13 & 0,15 \\
\hline Gha $\left(\mathrm{m}^{2} / \mathrm{ha}\right)$ & 3,89 & 43,26 & 21,65 & 8,73 & 1,25 & 34,87 & 17,64 & 7,67 & 1,17 & 51,82 & 20,69 & 10,15 & 0,81 & 36,80 & 17,91 & 9,18 \\
\hline $\operatorname{Gsup}\left(\mathrm{m}^{2} / \mathrm{ha}\right)$ & 0,00 & 36,94 & 8,99 & 8,33 & 0,00 & 30,54 & 11,03 & 8,85 & 0,00 & 37,02 & 9,70 & 10,50 & 0,00 & 32,31 & 7,55 & 7,60 \\
\hline Grel & 0,00 & 1,00 & 0,39 & 0,30 & 0,00 & 1,00 & 0,60 & 0,38 & 0,00 & 0,99 & 0,39 & 0,34 & 0,00 & 0,93 & 0,36 & 0,29 \\
\hline Gdist (m/ha) & 0,33 & 6,95 & 2,26 & 1,03 & 0,10 & 4,66 & 1,83 & 0,93 & 0,22 & 5,85 & 1,99 & 1,01 & 0,07 & 5,24 & 1,76 & 0,98 \\
\hline Elev (m) & 626,00 & 688,00 & 646,85 & 14,89 & 627,00 & 813,00 & 696,61 & 43,82 & 613,00 & 678,00 & 647,17 & 11,09 & 619,00 & 678,00 & 647,10 & 13,18 \\
\hline Flacc $\left(\mathrm{m}^{2}\right)$ & 0,00 & 24,00 & 2,49 & 4,48 & 0,00 & 17,00 & 1,17 & 2,65 & 0,00 & 67,00 & 2,57 & 9,61 & 0,00 & 63,00 & 2,68 & 9,25 \\
\hline$T P I$ & $-3,00$ & 3,60 & 0,44 & 1,57 & $-3,00$ & 4,60 & 0,59 & 1,62 & $-5,80$ & 6,00 & 0,59 & 1,71 & $-4,20$ & 5,20 & 0,52 & 1,80 \\
\hline$M C A\left(10^{3} \mathrm{~m}^{2}\right)$ & 23,79 & 156,45 & 56,07 & 17,56 & 15,56 & 209,54 & 83,34 & 53,42 & 19,61 & 151,82 & 63,54 & 25,94 & 25,56 & 126,64 & 64,24 & 25,39 \\
\hline SWI & 12,07 & 14,51 & 13,22 & 0,61 & 11,75 & 14,92 & 13,64 & 0,68 & 12,13 & 14,99 & 13,56 & 0,59 & 12,07 & 14,84 & 13,59 & 0,67 \\
\hline
\end{tabular}


Annexe 2.

Accroissements périodiques moyens ( $A M, \mathrm{~cm} / \mathrm{an})$ par essence (assamela, Pericopsis elata, moabi, Baillonella toxisperma, sapelli, Entandrophragma cylindricum, tali, Erythrophleum suaveolens) et par classe de diamètre (de 20 à plus de $100 \mathrm{~cm}$ ). Les valeurs indiquées en italique correspondent aux intervalles de confiance $(1-\alpha=95 \%)$. Le nombre d'arbres $(N)$ mesurés est indiqué par essence.

\begin{tabular}{|c|c|c|c|c|c|c|c|c|}
\hline \multirow[t]{2}{*}{ Classe de diamètre $(\mathrm{cm})$} & \multicolumn{2}{|c|}{$\begin{array}{c}\text { Assamela } \\
\text { Pericopsis elata }\end{array}$} & \multicolumn{2}{|c|}{$\begin{array}{c}\text { Moabi } \\
\text { Baillonella toxisperma }\end{array}$} & \multicolumn{2}{|c|}{$\begin{array}{c}\text { Sapelli } \\
\text { Entandrophragma cylindricum }\end{array}$} & \multicolumn{2}{|c|}{$\begin{array}{l}\text { Tali } \\
\text { Erythrophleum suaveolens }\end{array}$} \\
\hline & $\mathbf{N}$ & AM & N & AM & $\mathbf{N}$ & AM & $\mathbf{N}$ & AM \\
\hline $20-29$ & 1 & $0,03 \pm 0,00$ & 7 & $0,31 \pm 0,18$ & 13 & $0,18 \pm 0,13$ & 0 & l \\
\hline 30-39 & 9 & $0,36 \pm 0,16$ & 15 & $0,35 \pm 0,14$ & 9 & $0,28 \pm 0,12$ & 5 & $0,79 \pm 0,26$ \\
\hline 40-49 & 23 & $0,47 \pm 0,09$ & 12 & $0,77 \pm 0,21$ & 18 & $0,52 \pm 0,17$ & 8 & $1,14 \pm 0,29$ \\
\hline $50-59$ & 21 & $0,44 \pm 0,12$ & 3 & $1,24 \pm 0,43$ & 12 & $0,76 \pm 0,12$ & 13 & $0,78 \pm 0,22$ \\
\hline $60-69$ & 24 & $0,41 \pm 0,08$ & 2 & $1,19 \pm 0,22$ & 14 & $0,72 \pm 0,17$ & 11 & $0,43 \pm 0,12$ \\
\hline 70-79 & 20 & $0,45 \pm 0,08$ & 4 & $1,46 \pm 0,39$ & 11 & $0,63 \pm 0,20$ & 19 & $0,35 \pm 0,12$ \\
\hline $80-89$ & 19 & $0,43 \pm 0,05$ & 2 & $1,02 \pm 1,56$ & 13 & $0,62 \pm 0,22$ & 11 & $0,38 \pm 0,22$ \\
\hline 90-99 & 13 & $0,46 \pm 0,05$ & 4 & $0,56 \pm 0,32$ & 10 & $0,43 \pm 0,15$ & 1 & $0,20 \pm 0,00$ \\
\hline$>100$ & 6 & $0,51 \pm 0,04$ & 5 & $1,27 \pm 0,27$ & 24 & $0,43 \pm 0,09$ & 5 & $0,20 \pm 0,10$ \\
\hline Total & 136 & $0,43 \pm 0,03$ & 54 & $0,73 \pm 0,14$ & 124 & $0,51 \pm 0,06$ & 73 & $0,55 \pm 0,10$ \\
\hline
\end{tabular}

/ : valeur manquante.

Annexe 3.

Performance des modèles de croissance $M_{2}$ en fonction de la variable utilisée pour exprimer l'intensité de la compétition : nombre de paramètres du modèle ( $p)$, critère d'information d'Akaike (AIC), erreur moyenne (ME), écart-type résiduel (ECTr), coefficient de détermination ajusté ( $\left.R^{2} a d j\right)$. La performance du modèle $M_{1} b$ est donnée dans la première ligne du tableau à titre de comparaison.

\begin{tabular}{|l|c|c|c|c|c|}
\hline Variables & $\mathbf{p}$ & AIC & ME & ECTr & $\mathbf{R}^{2}$ adj \\
\hline Modèle M1b & & & & & \\
\hline- & 9 & 146,0 & $0,002 \pm 0,029$ & 0,288 & 0,341 \\
\hline Modèle M2 & & & & & \\
\hline Nha & 13 & 142,4 & $0,003 \pm 0,028$ & 0,285 & 0,354 \\
\hline Nsup & 13 & 98,0 & $0,003 \pm 0,027$ & 0,269 & 0,424 \\
\hline Nrel & 13 & 126,6 & $0,002 \pm 0,028$ & 0,280 & 0,380 \\
\hline Gha & 13 & 127,0 & $0,002 \pm 0,028$ & 0,280 & 0,379 \\
\hline Gsup & 13 & 112,5 & $0,002 \pm 0,027$ & 0,275 & 0,402 \\
\hline Grel & 13 & 124,3 & $0,002 \pm 0,028$ & 0,279 & 0,384 \\
\hline Gdist & 13 & 127,6 & $0,003 \pm 0,028$ & 0,280 & 0,378 \\
\hline Statut & 13 & 134,5 & $0,001 \pm 0,028$ & 0,282 & 0,367 \\
\hline Dawkins & 126,1 & $0,002 \pm 0,028$ & 0,279 & 0,381 \\
\hline
\end{tabular}

\section{Remerciements}

Les auteurs remercient la société Pallisco pour l'appui logistique dans la récolte des données et le financement de la recherche, Yves Brostaux et Anaïs Gorel pour leurs conseils dans l'analyse des données.

\section{Références bibliographiques}

Baker T. R., Swaine M. D., Burslem D. F., 2003. Variation in tropical forest growth rates: combined effects of functional group composition and resource availability. Perspectives in Plant Ecology, Evolution and Systematics, 6: 21-36.

Bayol N., Demarquez B., De Wasseige C., Atyi R. E., Fisher J.-F., Nasi R., Pasquier A., Rossi X., Steil M., Vivien C., 2012. Forest management and the timber sector in Central Africa. In: De Wasseige C., De Marcken P., Bayol N., Hiol Hiol F., Mayaux P., Desclée B., Nasi R., Billand A., Defourny P., Eba'a Atyi R. (Eds). The Forests of the Congo Basin - State of the Forest 2010. Luxembourg, Publications Office of the European Union, 43-62.

Beven K. J., Kirkby M. J., 1979. A physically based, variable contributing area model of basin hydrology. Hydrological Sciences Bulletin, 24: 43-69.

Boehner J., Koethe R., Conrad O., Gross J., Ringeler A., Selige T., 2002. Soil regionalisation by means of terrain analysis and process parameterisation. European Soil Bureau, Research Report $\mathrm{n}^{\circ} 7$.

Bourland N., Kouadio Y. L., Lejeune P., Sonké B., Philippart J., Daïnou K., Fétéké F., Doucet J.-L., 2012. Ecology of Pericopsis elata (Fabaceae), an endangered timber species in Southeastern Cameroon. Biotropica, 44 (6): 840-847. 
Dawkins H. C., 1958. The management of natural tropical high forest with special reference to Uganda. University of Oxford, Imperial Forestry Institute.

Debroux L., 1998. L'aménagement des forêts tropicales fondé sur la gestion des populations d'arbres : l'exemple du moabi dans la forêt du Dja (Cameroun). Thèse, Faculté universitaire des sciences agronomiques de Gembloux, Belgique, 285 p.

Détienne P., Oyono F., Durrieu de Madron L., Demarquez B., Nasi R., 1998. L'analyse des cernes : applications aux études de croissance de quelques essences en peuplements naturels de forêt dense africaine. Montpellier, France, Cirad-Forêt, série Forafri, 15, $36 \mathrm{p}$.

De Wasseige C., De Marcken P., Bayol N., Hiol Hiol F., Mayaux P., Desclée B., Nasi R., Billand A., Defourny P., Eba'a Atyi R. (éds), 2012. Les forêts du bassin du Congo - État des Forêts 2010. Luxembourg, Office des publications de l'Union européenne, $276 \mathrm{p}$.

Doucet J.-L., 2003. L'alliance délicate de la gestion et de la biodiversité dans les forêts du Gabon. Thèse, Faculté universitaire des sciences agronomiques de Gembloux, Belgique, 323 p.

Doucet J.-L., Kouadio Y. L., Monticelli D., Lejeune P., 2009. Enrichment of logging gaps with moabi (Baillonella toxisperma Pierre) in a Central African rain forest. Forest Ecology and Management, 258: 2407-2415.

Dufour-Kowalski S., Courbaud B., Dreyfus P., Meredieu C., de Coligny F., 2012. Capsis: an open software framework and community for forest growth modelling. Annals of Forest Science, 69: 221-233.

Duminil J., Heuertz M., Doucet J.-L., Bourland N., Cruaud C., Gavory F., Doumenge C., Navascués M., Hardy O. J., 2010. CpDNA-based species identification and phylogeography: application to African tropical tree species. Molecular Ecology, 19 (24): 5469-5483.

Durrieu de Madron L., Forni E., 1997. Aménagement forestier dans l'Est du Cameroun : Structure du peuplement et périodicité d'exploitation. Bois et Forêts des Tropiques, 254 : 39-50. [En ligne] http://bft.cirad.fr/cd/BFT_254_39-50.pdf

Durrieu de Madron L., Nasi R., Détienne P., 2000. Accroissements diamétriques de quelques essences en forêt dense africaine. Bois et Forêts des Tropiques, $263: 63-74$. [En ligne] http://bft.cirad.fr/cd/BFT_263_63-74.pdf

Fargeot C., Forni E., Nasi R., 2004. Réflexions sur l'aménagement des forêts de production dans le bassin du Congo. Bois et Forêts des Tropiques, $281: 19-34$.

[En ligne] http://bft.cirad.fr/cd/BFT_281_19-34.pdf

Fayolle A., Rondeux J., Doucet J.-L., Ernst G., Bouissou C., Quevauvillers S., Bourland N., Fétéké R., Lejeune P., 2013. Réviser les tarifs de cubage pour mieux gérer les forêts du Cameroun. Bois et Forêts des Tropiques. 317 : 35-49.

[En ligne] http://bft.cirad.fr/cd/BFT_317_35-49.pdf

Gourlet-Fleury S., 1998. Indices de compétition en forêt dense tropicale humide : étude de cas sur le dispositif sylvicole expérimental de Paracou (Guyane française). Annales des Sciences Forestières, 55 (6) : 623-654.

Gourlet-Fleury S., Houllier F., 2000. Modelling diameter increment in a lowland evergreen rain forest in French Guiana. Forest Ecology and Management, 131: 269-289.
Gourlet-Fleury S., Mortier F., Fayolle A., Baya F., Ouédraogo D., Bénédet F., Picard N., 2013. Tropical forest recovery from logging: a 24 year silvicultural experiment from Central Africa. Philosophical Transactions of the Royal Society B: Biological Sciences, 368 (1625), 10 p.

Hall J. B., Swaine M. D., 1981. Distribution and ecology of vascular plants in a tropical rain forest. La Haye, Pays-Bas, W. Junk Publishers, $383 \mathrm{p}$.

Hall J. S., McKenna J. J., Ashton P. M. S., Gregoire T. G., 2004. Habitat characterizations underestimate the role of edaphic factors controlling the distribution of Entandrophragma. Ecology, 85: 2171-2183.

Hawthorne W. D., 1995. Ecological profiles of Ghanaian forest trees. Oxford Forestry Institute, Department of Plant Sciences, University of Oxford, Tropical Forestry Papers, 29, 345 p.

Hérault B., Bachelot B., Poorter L., Rossi V., Bongers F., Chave J., Paine C. E. T., Wagner F., Baraloto C., 2011. Functional traits shape ontogenetic growth trajectories of rain forest tree species. Journal of Ecology, 99: 1431-1440.

Kouadio Y. L., Neuba D. F. R., Koné M., Bourland N., Doucet J.-L., 2014. Étude de la croissance diamétrique de quatre essences de bois d'œuvre exploitées à l'Est du Cameroun. Journal of Applied Biosciences, 77 : 6444-6455.

Ledermann T., Eckmüllner O., 2004. A method to attain uniform resolution of the competition variable Basal-Area-inLarger Trees (BAL) during forest growth projections of small plots. Ecological Modelling, 171: 195-206.

Lejeune P., 1996. Développement d'un modèle de type arbre appliqué à la croissance des peuplements feuillus irréguliers du sud de la Belgique. Canadian Journal of Forest Research, $26: 1838-1848$.

Letouzey R., 1985. Notice de la carte phytogéographique du Cameroun au 1/500 000. Orohydrographie. Toulouse, France, Institut de la carte internationale de la végétation, $63 \mathrm{p}$.

Moravie M. A., Pascal J., Auger P., 1997. Investigating canopy regeneration processes through individual-based spatial models: application to a tropical rain forest. Ecological Modelling, 104: 241-260.

Novella N. S., Thiaw W. M., 2012. African Rainfall Climatology Version 2 for Famine Early Warning Systems. Journal of Applied Meteorology and Climatology, 52: 588-606.

Obiang N. L. E., Ngomanda A., White L. J. T., Jeffery K. J., Chézeaux E., Picard N., 2013. Disentangling the effect of size and competition: a growth model for Aucoumea klaineana. Annals of Forest Science, 70: 241-249.

Obiang N. L. E., Ngomanda A., White L. J. T., Jeffery K. J., Chézeaux E., Picard N., 2012. A growth model for azobé, Lophira alata, in Gabon. Bois et Forêts des Tropiques, 314 4: 65-72. [En ligne] http://bft.cirad.fr/cd/BFT_314_65-72.pdf

Picard N., Gourlet-Fleury S., Forni E., 2012. Stock recovery rates are not the panacea to assess timber yield sustainability: Evidence from managed Central African forests. Forest Ecology and Management, 281: 12-22.

Picard N., Yalibanda Y., Namkosserena S., Baya F., 2008. Estimating the stock recovery rate using matrix models. Forest Ecology and Management, 255: 3597-3605. 
Pinheiro J., Bates D., DebRoy S., Sarkar D., R Development Core Team, 2013. nlme: Linear and Nonlinear Mixed Effects Models. R package version 3.1-111. Vienne, Autriche, R Foundation for Statistical Computing.

Pokharel B., Dech J. P., 2012. Mixed-effects basal area increment models for tree species in the boreal forest of Ontario, Canada using an ecological land classification approach to incorporate site effects. Forestry, 85: 255-270.

R Development Core Team, 2013. R: A language and environment for statistical computing. Vienne, Autriche, R Foundation for Statistical Computing.

Reuter H. I., Nelson A., Jarvis A., 2007. An evaluation of voidfilling interpolation methods for SRTM data. International Journal of Geographical Information Science, 21: 983-1008.

Steneker G. A., Jarvis J. M., 1963. A preliminary study to assess competition in a white spruce - trembling aspen stand. The Forestry Chronicle, 39: 334-336.

Therrell M. D., Stahle D. W., Mukelabai M. M., Shugart H. H., 2007. Age, and radial growth dynamics of Pterocarpus angolensis in southern Africa. Forest Ecology and Management, 244: $24-31$.
Uriarte M., Condit R., Canham C. D., Hubbell S. P., 2004. A spatially explicit model of sapling growth in a tropical forest: does the identity of neighbors matter? Journal of Ecology, 92: 348-360.

Weiner J., Thomas S. C., 2001. The Nature of Tree Growth and the "Age-Related Decline in Forest Productivity". Oikos, 94: 374-376.

WRI (World Resources Institute), 2009. Atlas forestier interactif du Gabon. Version pilote. Document de synthèse. Washington D.C., USA, WRI, MEFEPA, 70 p.

Zanne A. E., Lopez-Gonzalez G., Coomes D.A., Ilic J., Jansen S., Lewis S. L., Miller R. B., Swenson N. G., Wiemann M. C., Chave J., 2009. Global wood density database. Data from: Towards a worldwide wood economics spectrum. Dryad Digital Repository.

[Online] http://dx.doi.org/10.5061/dryad.234

Zhao D., Borders B., Wilson M., 2004. Individual-tree diameter growth and mortality models for bottomland mixed-species hardwood stands in the lower Mississippi alluvial valley. Forest Ecology and Management, 199: 307-322. 